\title{
EU-Ausländer in der Praxis? So wird's gemacht!
}

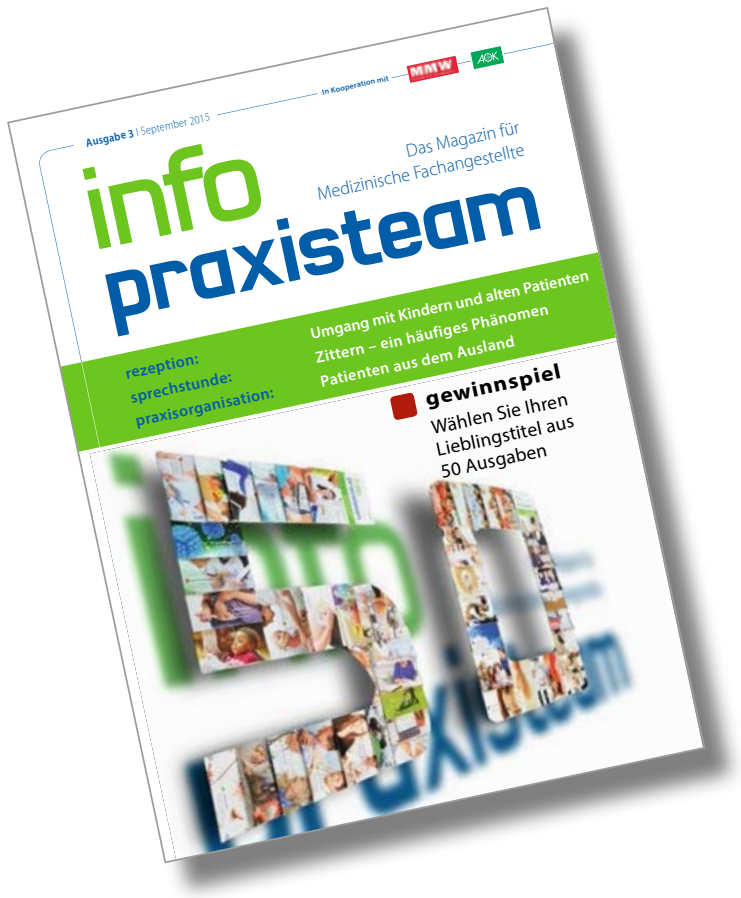

Dieser Ausgabe von MMW liegt wieder „info praxisteam“ bei, das Magazin für Medizinische Fachangestellte. Thema in Heft 3/15 ist der Umgang mit Touristen in der Praxis. Ausländer aus der Europäischen Union können ohne große Umstände auf Kasse behandelt werden - bei anderen Staaten wird es schwieriger.

— Für Patienten aus dem Ausland, die als Touristen hier in Deutschland unterwegs sind, gibt es klare Vorgaben für die medizinische Versorgung. Am einfachsten ist die Vorlage der Europäischen Krankenversichertenkarte (EHIC) oder einer Ersatzbescheinigung (PEB). Über eine EHIC verfügen in der Regel Touristen aus anderen EU-Ländern wie auch aus den assoziierten Staaten Island, Liechtenstein, Norwegen und der Schweiz.

Sie haben Anspruch auf alle Leistungen, die sich während ihres Aufenthalts in
Deutschland als medizinisch notwendig erweisen. Das betrifft die unmittelbar erforderliche medizinische Versorgung, aber auch eine fortlaufende Versorgung für chronisch Kranke, etwa Dialysepatienten.

Das Praxisteam überprüft in diesem Fall die Identität des Patienten anhand seines Ausweises. Karte und Identitätsnachweis müssen je zweimal beidseitig fotokopiert werden - einmal für die Krankenkasse und einmal zum Aufbewahren in der Praxis. Für diese Fotokopien ist jeweils die EBM-Ziffer 40144 berechnungsfähig. Dazu müssen die Muster 80 und 81 ausgefüllt werden, Letzteres vom Patienten selbst. Der Leistungsanspruch richtet sich nach der voraussichtlichen Aufenthaltsdauer. Bei der Verordnung muss darauf geachtet werden, dass die Packungsgrößen entsprechend der Aufenthaltsdauer gewählt werden.

\section{Abrechnung qua Ersatzverfahren}

Sie können die Kosten für die Behandlung nach den Regelungen des Ersatzverfahrens abrechnen. Dazu stellen Sie einen Abrechnungsschein (Muster 5) aus. In das Adressfeld tragen Sie Name, Vornamen und Geburtsdatum sowie die gewählte Krankenkasse ein. In das Feld "Status" fügen Sie bei "Versichertenart" eine 1 und bei „Besondere Personengruppe" eine 7 ein. Dadurch laufen diese Abrechnungen außerhalb des normalen Budgets - und bedeuten somit bares Geld für die Praxis. Die gesammelten Dokumente - also Kopien der EHIC und des Ausweises sowie die ausgefüllten und unterschriebenen Muster 80 und 81 - schicken Sie noch am gleichen Tag zur gewählten Krankenkasse.

Patienten, die auf Basis eines bilateralen Abkommens über Soziale Sicherheit Anspruch auf Leistungen bei Krankheit haben, können bei einem Aufenthalt in Deutschland ebenfalls ärztliche Hilfe beanspruchen.
Diese Patienten müssen sich mit dem Anspruchsnachweis ihrer heimischen Krankenkasse zunächst an eine deutsche Krankenkasse wenden, die dann einen Abrechnungsschein ausstellt. Eine Überweisung zu einem weiteren Arzt ist nicht möglich. Wenn Sie die Notwendigkeit anderweitiger ärztlicher Behandlung bescheinigen (Muster 16), bekommt der Patient bei der Kasse einen weiteren Abrechnungsschein. Solche bilateralen Abkommen gibt es mit Bosnien-Herzegowina, Mazedonien, Montenegro, Serbien, der Türkei und Tunesien. Patienten aus anderen Ländern, etwa aus Amerika oder Asien, können nur gegen Privatrechnung werden.

In der Rubrik „rezeption“ geht es unter anderem um den Umgang mit Kindern, Jugendlichen und alten Patienten. Und in der "sprechstunde" werden verschiedene Arten des Tremors vorgestellt und die therapeutischen Kosequenzen diskutiert.

Dr. Reinhard Merz .

\section{Web-Tipp}

„info praxisteam" erscheint sechsmal jährlich. Alle Beiträge sind unter www.info-praxisteam.de auch im Internet abrufbar und können direkt kommentiert werden.

Auch für mobile Endgeräte wie Smartphones oder Tablets ist „info praxisteam" verfügbar. Die App wird sowohl für iOS von Apple als auch für Geräte mit Android-Plattform angeboten. Mehr darüber, wie man die App beziehen kann, erfahren Sie auf der oben angegebenen Website.

Zu den Beiträgen im Heft gibt es von der Redaktion ausgesuchte Verweise auf weiterführende Informationen im Internet. Viele davon sind auch für Sie als Arzt interessant.

Sie erreichen uns per E-Mail:

redaktion@info-praxisteam.de 